\title{
Viscoelastic Phase Patterning in Artificial Protein Hydrogels
}

Peter Rapp, Bradley Silverman

Submitted date: 18/11/2020 Posted date: 19/11/2020

Licence: CC BY-NC-ND 4.0

Citation information: Rapp, Peter; Silverman, Bradley (2020): Viscoelastic Phase Patterning in Artificial Protein Hydrogels. ChemRxiv. Preprint. https://doi.org/10.26434/chemrxiv.13250102.v1

Viscoelastic forces can affect the dynamics of pattern formation during phase separation in polymeric materials. We programmed an artificial protein hydrogel to undergo viscoelastic phase separation above a critical temperature. Highly dynamic phase patterns that coarsened under the influence of viscoelastic stresses spontaneously emerged in these gels. Local oxidative crosslinking promoted by mild photobleaching could be used to initiate phase separation locally, enabling the creation of non-equilibrium patterns that evolved under the influence of surface tension and viscoelastic stresses to yield dynamic structures of controlled size and shape.

File list (6)

rapp-silverman-phasepatterning-chemRxiv-201118.pdf (1.55 MiB)

rapp-silverman-phasepatterning-chemRxiv-supp.pdf (459.32 KiB)

movieS1.mov (2.46 MiB)

movieS2.mov (9.87 MiB)

movieS3.mov (97.45 KiB)

movieS4.mov (7.03 MiB) view on ChemRxiv - download file view on ChemRxiv • download file view on ChemRxiv • download file view on ChemRxiv • download file view on ChemRxiv • download file view on ChemRxiv • download file 


\title{
Title: Viscoelastic phase patterning in artificial protein hydrogels
}

\author{
Authors: Peter B. Rapp*†, Bradley R. Silverman
}

\begin{abstract}
Affiliations:
Division of Chemistry and Chemical Engineering, California Institute of Technology, Pasadena, CA 91125, USA.

*Correspondence to: peter.rapp@yale.edu

†Present address: Department of Chemistry, Yale University, 225 Prospect St., New Haven, CT 06511
\end{abstract}

\begin{abstract}
Viscoelastic forces can affect the dynamics of pattern formation during phase separation in polymeric materials. We programmed an artificial protein hydrogel to undergo viscoelastic phase separation above a critical temperature. Highly dynamic phase patterns that coarsened under the influence of viscoelastic stresses spontaneously emerged in these gels. Local oxidative crosslinking promoted by mild photobleaching could be used to initiate phase separation locally, enabling the creation of non-equilibrium patterns that evolved under the influence of surface tension and viscoelastic stresses to yield dynamic structures of controlled size and shape.
\end{abstract}

One Sentence Summary: Viscoelastic stress and surface tension control pattern development during phase separation in artificial protein hydrogels. 
Main Text: Phase separation can induce spontaneous pattern formation in polymeric materials (1). This provides a simple way to control material microstructure, enabling access to diverse and useful material properties (2-4). Simple solids and liquids develop either bicontinuous or droplet patterns in transition to binary equilibrium states (5). In contrast, web- and sponge-like patterns can emerge in viscoelastic materials, which have dynamic properties intermediate between fluids and solids (5-7). Whereas pattern growth in simple mixtures is often considered to be scale invariant or "self-similar" (8), stress relaxation in viscoelastic materials can break the selfsimilarity or scale invariance of developing patterns $(9,10)$.

Viscoelastic effects on phase separation have been recognized in various contexts $(6,9$, 11-14), but remain difficult to predict and control. We hypothesized that artificial proteins could be programmed to undergo viscoelastic phase separation by encoding them with two key properties: $i$ ) the ability to assemble into associative networks that both store and dissipate mechanical stress and ii) a thermal phase transition that could be triggered directly from the reversibly crosslinked state. We recently cloned and recombinantly expressed an artificial protein polymer that satisfies these two design criteria (Fig. 1A and Table S1) $(15,16)$. The polymer, which is designated "PEP", comprises a flexible, water-soluble "E" midblock consisting of 30 repeats of the elastin-like pentapeptide sequence $(\mathrm{VPGXG})_{n}$, where $\mathrm{X}$ is either Val or Glu. This domain is flanked by two identical "P" endblocks that spontaneously self-associate into homopentameric coiled-coil bundles (17). Self-association of the PEP endblocks promotes reversible self-assembly of the protein into a viscoelastic network (Fig. 1A and fig. S1) (16, 18, 19). The midblock simultaneously confers tunable phase behavior on these networks, owing to phase separation above a predictable lower critical solution temperature (LCST) that is a wellknown property of elastins (20-22). 


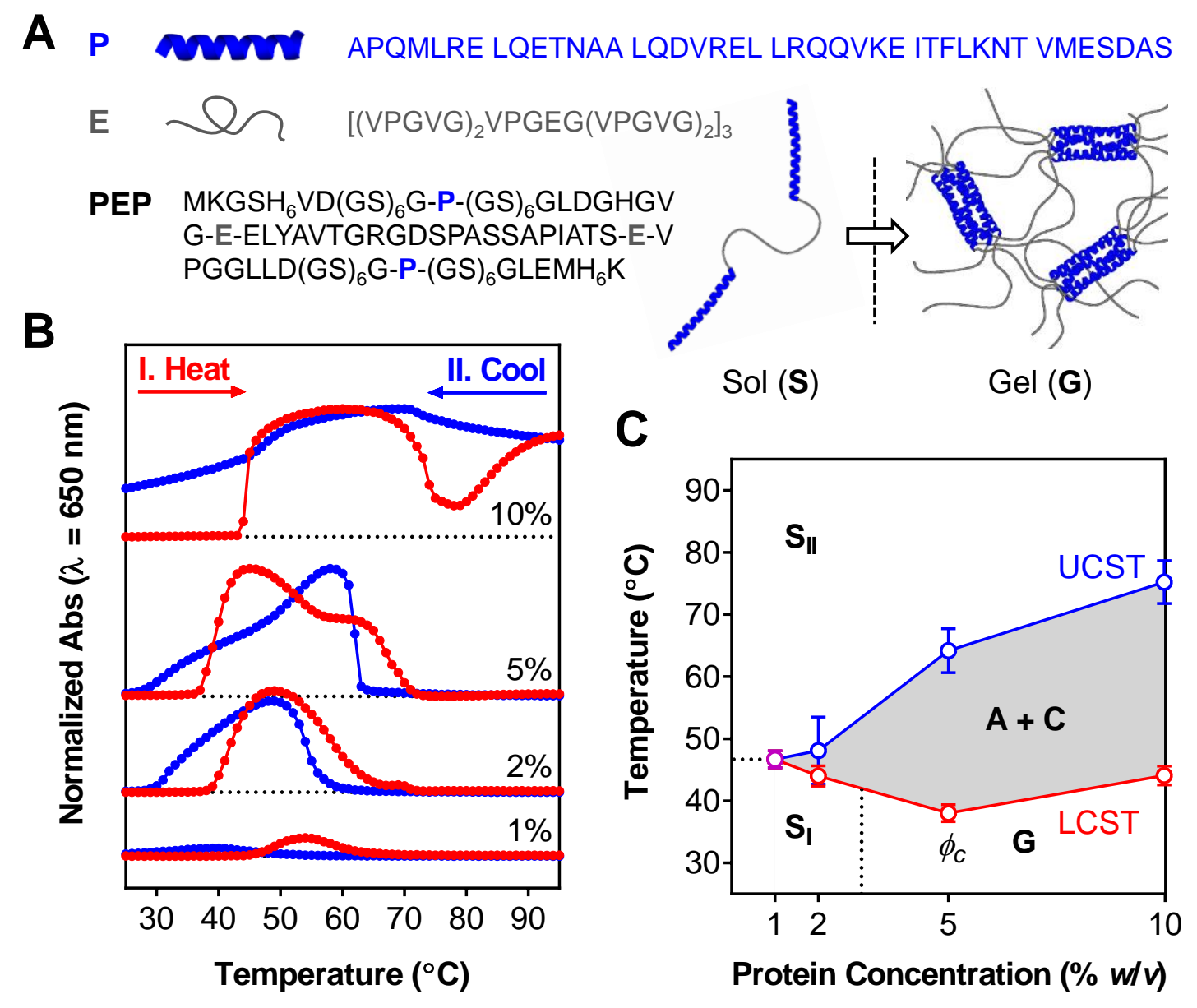

Fig. 1. Sequence programmable phase separation in an artificial protein hydrogel. (A) Sequence of PEP. The protein comprises two coiled-coil "P" endblocks which flank a water-soluble elastin-like "E" midblock. When swollen in aqueous buffer, oligomerization of the endblocks drives reversible assembly into a viscoelastic gel. (B) Cloud-point measurements on PEP gels ( $\mathrm{pH}$ 6.5) reveal phase separation and reentrant behavior. The gels were first heated then cooled over the range $25{ }^{\circ} \mathrm{C}$ to $95{ }^{\circ} \mathrm{C}$ at a rate of $3{ }^{\circ} \mathrm{C} \mathrm{min}{ }^{-1}$. Gel turbidity was monitored at $650 \mathrm{~nm}$. (C) The $T-\varphi$ phase diagram of PEP contains an immiscibility loop: $\mathrm{S}_{\mathrm{I}}$, sol phase; G, reversible gel; $A+C$, aqueous phase (protein-poor) and coacervate phase (protein-rich); $S_{\text {II }}$, reentrant sol phase. Points represent mean $\pm \mathrm{SD}(n=4)$.

On the basis of the midblock sequence, we predicted that PEP networks would exhibit LCST-type transitions at about $70{ }^{\circ} \mathrm{C}$ (22). Surprisingly, cloud-point measurements revealed 
phase separation at a much lower temperature: at $38{ }^{\circ} \mathrm{C}(\mathrm{LCST}), 5 \%(w / v)$ PEP gels separated into aqueous (A) and protein-rich (C) coacervate phases (Fig. 1B). Moreover, the gels exhibited reentrant behavior: continued heating above $64{ }^{\circ} \mathrm{C}$ completely restored protein miscibility, and a second cloud-point (UCST) became evident upon subsequent cooling (23). Similar measurements at other concentrations revealed an immiscibility loop in the $T-\varphi$ phase diagram and an apparent critical composition near 5\% ( $\phi_{c}$, Fig. 1C). Moreover, 10\% gels appeared to exhibit a third cloudpoint above $80{ }^{\circ} \mathrm{C}$. Phase diagrams of this general class are rare (24-28), and are diagnostic of a unique temperature dependence for the Flory polymer-solvent interaction parameter $\chi(27)$.

In an effort to understand this phase behavior, we performed cloud-point measurements on two additional engineered proteins (Table S1). Concentrated solutions of an uncrosslinked "E" protein (which lacks the associative $\mathrm{P}$ domains of PEP) underwent phase separation near $85{ }^{\circ} \mathrm{C}$, closer to the LCST predicted from sequence (Fig. S2). Using site-directed mutagenesis, we installed a single cysteine in this protein to promote disulfide crosslinking under oxidizing conditions. Solutions of a disulfide dimerized "EC" protein exhibited an LCST at $63{ }^{\circ} \mathrm{C}$ (Fig. S2), consistent with previous observations that noncovalent interchain association can reduce the transition temperatures of elastins (24). We therefore attribute the depressed LCST of PEP gels to the highly multimeric nature of the reversibly crosslinked state, and the subsequent reentrant behavior at the UCST to gradual thermal unfolding of the $\mathrm{P}$ endblocks between $25{ }^{\circ} \mathrm{C}$ and $65{ }^{\circ} \mathrm{C}$ (29). Unfolding was evident from the mechanical softening of gels during heating (Fig. S1).

Next, we labeled PEP networks with fluorescent probe chains (designated PE $\mathrm{P}-f m$, Fig. S3) in order to visualize pattern evolution during phase separation above the LCST. Following fast heating to $50{ }^{\circ} \mathrm{C}$ (heating rate $\sim 0.4{ }^{\circ} \mathrm{C} / \mathrm{s}$ ), phase boundaries formed and the gel began to shrink. 

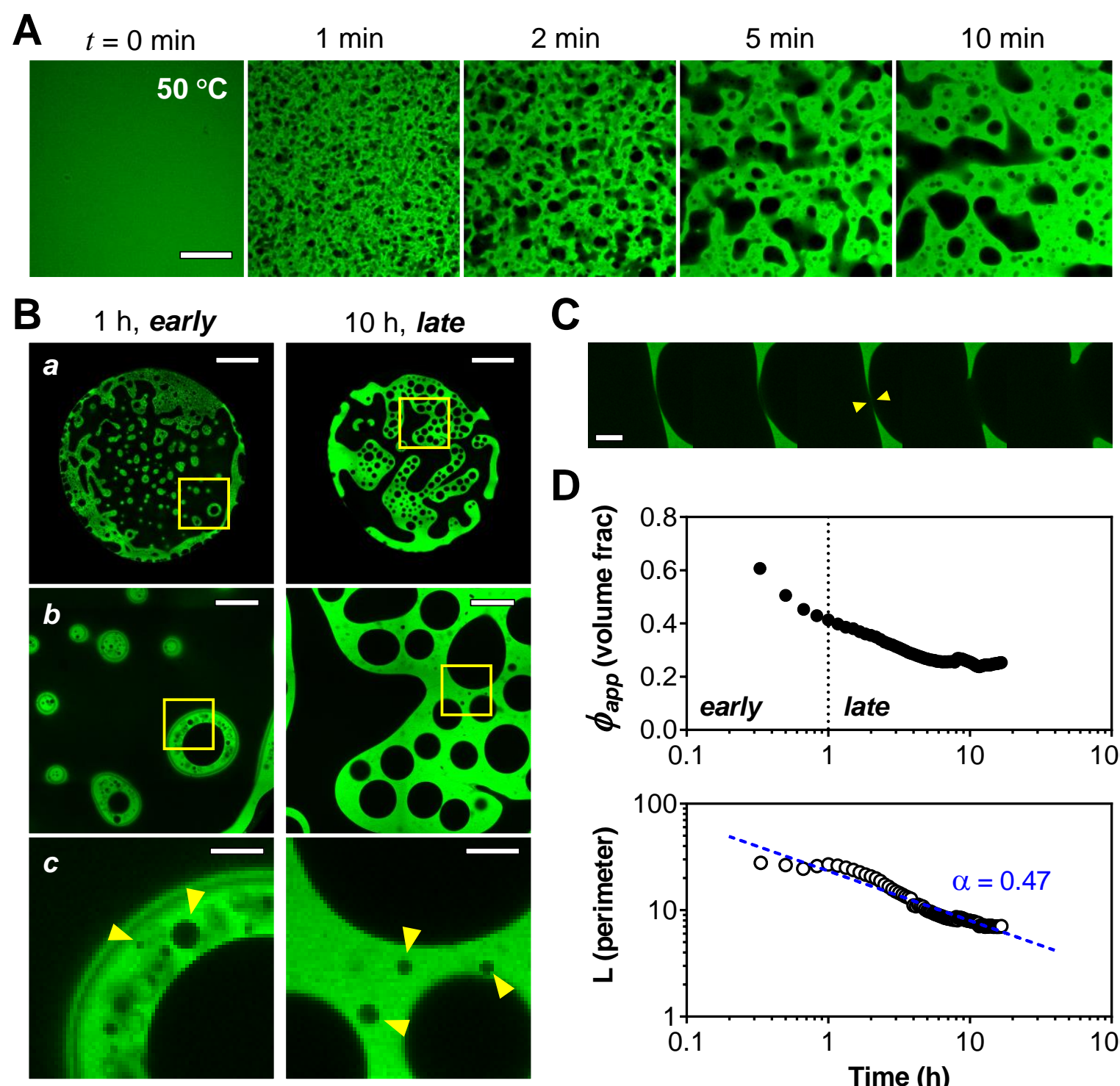

D
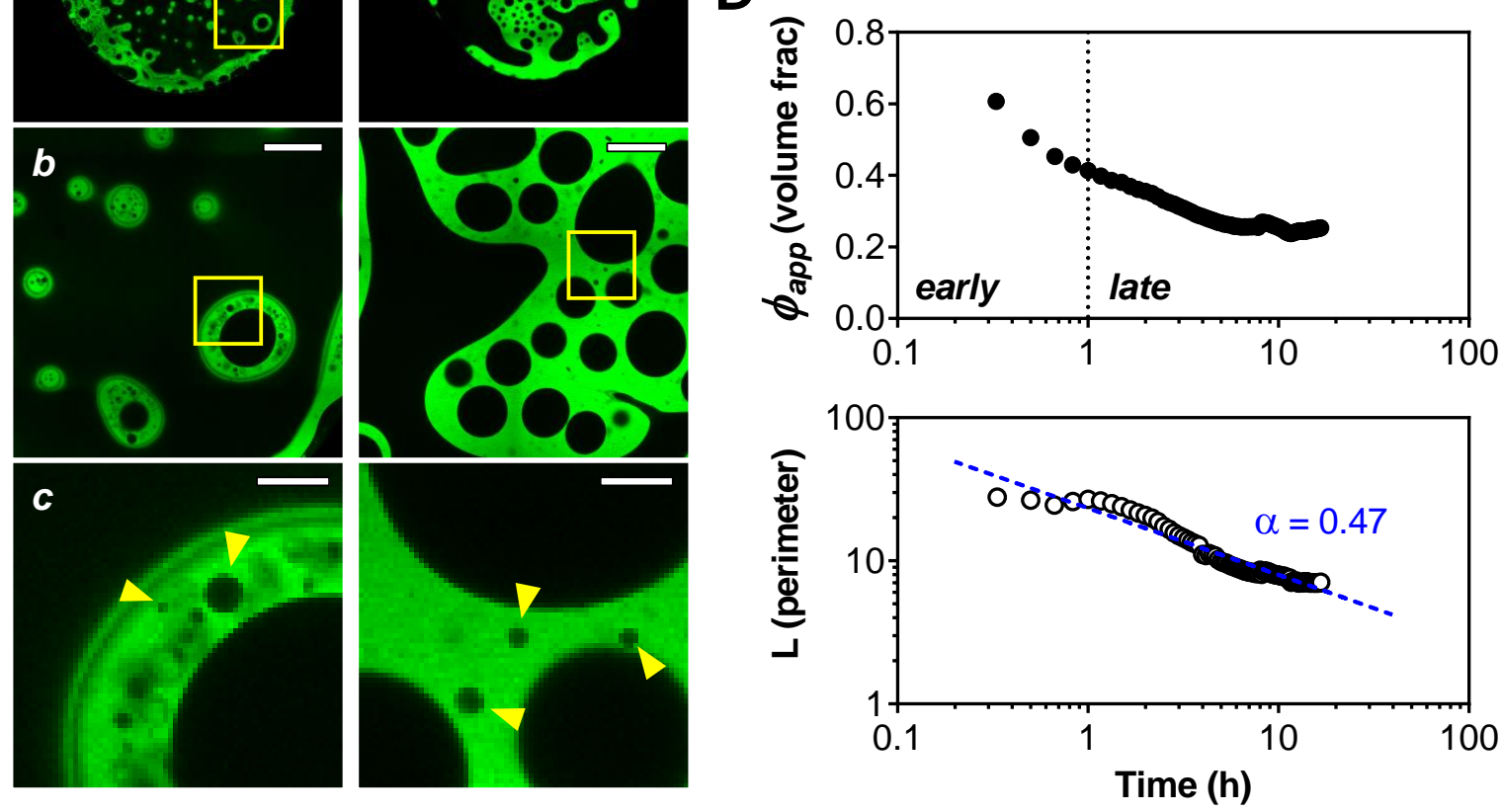

Fig. 2. Viscoelastic phase separation in PEP hydrogels. (A) Rapid heating of $10 \%$ PEP gels to $50{ }^{\circ} \mathrm{C}$ promotes emergence of a transient sponge-like phase within the first $10 \mathrm{~min}$. Scale bar $=100 \mu \mathrm{m}$. (B) Overview images of early$(<1 \mathrm{~h})$ and late- $(10 \mathrm{~h})$ stage coarsening at several magnification levels suggest the absence of a characteristic length scale and the operation of multiple coarsening modes. Scale bars: (a) $1 \mathrm{~mm},($ b) $200 \mu \mathrm{m}$, (c) $50 \mu \mathrm{m}$. (C) Viscoelastic "breakage" events relaxed local domain configuration during the late stage (shown are 5 frames taken over 10 min, after $8 \mathrm{~h}$ of heating). Scale bar $=50 \mu \mathrm{m}$. (D) Quantitative analysis of pattern evolution. (Top) The apparent volume fraction of the protein-rich coacervate phase decreased well into the late stage. (Bottom) The interfacial line density (normalized total perimeter) obeyed an approximate power law of the form $L \sim t^{-\alpha}(\alpha=0.4 \pm 0.1, n=4)$. 
The developing coacervate (protein-rich) phase initially resisted collapse, generating a transient, elastic restoring force that caused dramatic emergence of a highly interconnected and sponge-like structure that began to condense as the stress relaxed (Fig. 2A and Movie S1). Remarkably, slow coarsening by aqueous droplet coalescence completely reconfigured this early-stage pattern over a period of several hours (Fig. 2B and Movie S2). The resulting late-stage pattern appeared to lack any characteristic length scale, as new aqueous droplets could still be seen nucleating within the coacervate even after many hours of heating (Fig. 2B, c).

This kind of pattern evolution is a hallmark of phase separation under the influence of viscoelastic stress (30). Tanaka has proposed that such web- and sponge-like patterns arise whenever the phase separation rate is faster than the internal stress relaxation rate of one of the phases $(6,31)$. Under these circumstances, early domain shapes evolve to satisfy a mechanical force balance. Subsequent viscoelastic stress relaxation generates elastic instabilities that promote domain breakage. Simultaneously, regular coarsening modes (e.g., droplet ripening) drive the domains into circular architectures to minimize interfacial tension. The operation of multiple coarsening modes, each dominating at different times, breaks the self-similarity of the phase decomposition, producing irregular features characterized by different length scales $(9,10,31)$. Our results are in qualitative agreement with this picture.

Several additional features of late stage pattern evolution support the persistence of a viscoelastic coarsening mode. We routinely observed events lasting several hours, in which slender coacervate tendrils "snapped" back to relax internal stress (Fig. 2C and Movie S3). The apparent volume fraction of the condensed coacervate phase obtained by image thresholding analysis decreased well into the late stage, implying a slowly increasing polymer concentration in this phase (Fig. 2D). According to Tanaka, such concentration change violates a prerequisite for so-called 
"self-similar" pattern evolution and domain growth, wherein consecutive configurations of the phase domain boundaries are geometrically similar throughout the entire coarsening process $(8$, 9). The striking difference between the early and late stage phase patterns (cf. Fig. 2A and 2B) is consistent with an absence of self-similar domain grown. Finally, the total interfacial perimeter decayed following an approximate power law $(\alpha=0.4 \pm 0.1$, Fig. 2D). This exponent suggests late-stage growth dominated by either droplet ripening or fusion $(\alpha=1 / 3)(31,32)$. Both growth mechanisms were clearly discernible by time-lapse microscopy (Movie S2).

We considered that our fluorescence-based phase visualization strategy might lend itself to directly quantifying chain diffusion during phase separation via fluorescence recovery after photobleaching (FRAP). The FRAP method has been widely used to characterize macromolecular transport in hydrogels $(33,34)$, and we recently applied it to study the mechanism of chain migration in PEP networks below the LCST $(19,35)$. Consistent with our previous study, gels labeled with the associative probe $\mathrm{PE}_{\mathrm{C}} \mathrm{P}-\mathrm{fm}$ (fig. S3) showed steady fluorescence recovery at $25^{\circ} \mathrm{C}$, implying normal diffusive chain migration through the network (Fig. 3A).

In contrast to this simple diffusive behavior, the fluorescence recovery behavior of gels photobleached near the LCST was highly anomalous. During phase separation onset at $40{ }^{\circ} \mathrm{C}$, photobleaching clearly perturbed the local phase domain structure, causing formation of thin coacervate spines around the bleach spot (arrowheads, Fig. 3A). The rate of fluorescence recovery during this early stage appeared accelerated relative to the late stage (Fig. 3B). Moreover, both the early- and late-stage recovery profiles were poorly fit by a standard FRAP model that attributes fluorescence recovery to simple (effective Fickian) diffusion (Fig. S4) (36).

To explore the origin of the anomalous FRAP traces, we performed photobleaching experiments with two spectrally independent probes. Photobleaching of a green probe $\left(\mathrm{PE}_{C} \mathrm{P}-f m\right.$, 


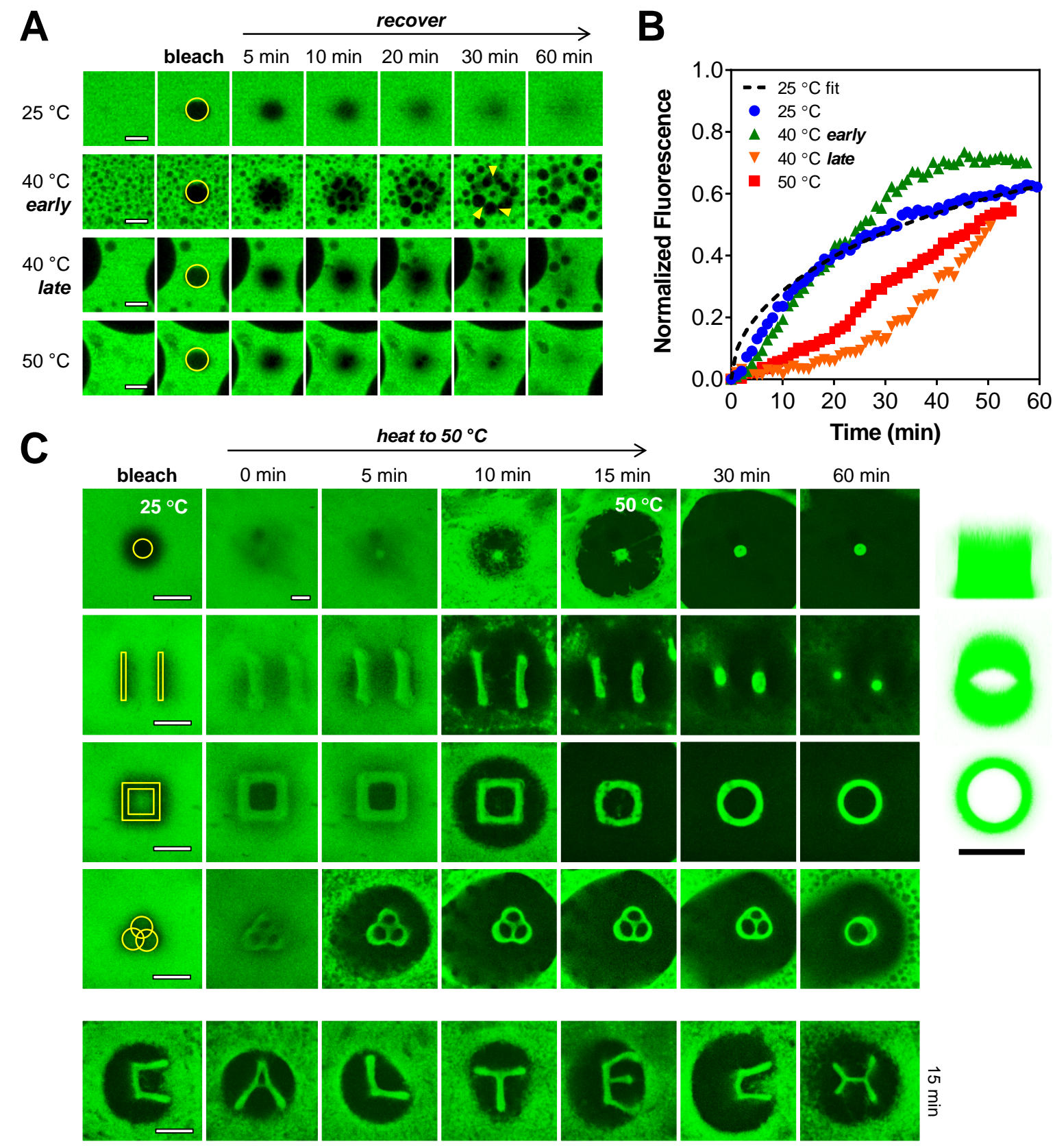

Fig. 3. Photobleaching enables patterning of phase-separated protein gels. (A) Fluorescence recovery after photobleaching (FRAP) was monitored for $60 \mathrm{~min}$ in $10 \%$ gels at temperatures below $\left(25^{\circ} \mathrm{C}\right)$ and above $\left(\geq 40{ }^{\circ} \mathrm{C}\right)$ the phase transition temperature (LCST). Photobleaching perturbed local domain structure during the fluorescence recovery period in gels recently heated to this temperature. Thin coacervate spines appeared around "early-stage" bleach spots $\left(40^{\circ} \mathrm{C}\right.$ early, $30 \mathrm{~min}$; heating began $10 \mathrm{~min}$ prior to photobleaching), but were absent from around "latestage" spots within gels bleached after $>10 \mathrm{~h}$ of coarsening $\left(40^{\circ} \mathrm{C}\right.$ late and $\left.50^{\circ} \mathrm{C}\right)$. Scale bars $=20 \mu \mathrm{m}$. (B) Quantitative fluorescence recovery data obtained by image analysis (traces correspond to panel $3 \mathrm{~A}$ ). Fluorescence recovery in 
phase-separated samples $\left(\geq 40^{\circ} \mathrm{C}\right)$ is anomalous and poorly fit by an effective diffusion model, whereas the recovery behavior at $25{ }^{\circ} \mathrm{C}$ is well fit (dashed line). (C) Heating of 5\% gels after photobleaching matured the photobleached regions into dynamic coacervate domains. The patterned domains evolved under the control of interfacial tension and a quickly relaxing mechanical stress, gradually producing cylindrical and droplet phase architectures that were stable for up to several hours. Scale bars $=100 \mu \mathrm{m}$.

490Ex/525Em) unexpectedly triggered diffusion of an unbleached red probe (PE $\mathrm{P}-\mathrm{trm}$, 596Ex/615Em) into the bleach spot (Fig. S3A). Fluorescein and rhodamine-based dyes are well known generators of singlet oxygen $(37,38)$, a highly reactive species that rapidly crosslinks proteins (39-41). We confirmed by electrophoretic analysis that irradiation of fluorescently labeled gels promoted mild oxidative crosslinking of PEP chains (Fig. S3B). Both crosslinking and bleach spot enrichment of the red probe were suppressed by sodium azide, a strong singlet oxygen quencher. Taken together, these results implicate mild oxidative crosslinking by photobleaching as the cause of the anomalous FRAP traces. Moreover, as the cloud-point measurements confirmed (Fig. S2), such crosslinking can depress the LCST of elastin chains (20). Apparently, local crosslinking of the network in our photobleaching experiments is sufficient to depress the LCST and trigger a local phase change, with subsequent chain enrichment in the bleach spot driven by equalization of the chemical potential at the bleach spot boundary.

Photobleaching prior to the onset of thermal phase separation had a striking effect on the local phase domain morphology (Fig. 3C). Samples bleached at $25{ }^{\circ} \mathrm{C}$ and then heated to $50{ }^{\circ} \mathrm{C}$ showed evidence of enrichment of chains in the bleached region. Rapid contraction of the coacervate above the network LCST then caused viscoelastic tearing around the bleach spot, which isolated the photobleached patterns in a wide depletion zone (Movie S4). During this period, the patterns behaved as soft viscoelastic bodies that were simultaneously stretched and rounded by the combined action of mechanical stress and surface tension. This interplay of coarsening 
mechanisms tended to create non-equilibrium coacervate structures exhibiting mild distension and pattern bending (e.g., the distortion of a photobleached " $\mathrm{H}$ " character). Ongoing stress relaxation and volume shrinking subsequently collapsed the patterns into symmetrical droplet and cylindrical structures that were gradually absorbed by the global coacervate after a period of several hours.

The experiments described here provide a clear demonstration of viscoelastic phase separation in artificial protein networks. The ability of such networks to both store and dissipate mechanical stress induces unusual sponge-like patterns and causes a breakdown in the selfsimilarity of the phase decomposition. Pattern evolution in such systems is governed by a balance of surface tension and viscoelastic stress. In our system, mild oxidative crosslinking could be used to influence the size and shape of the phase separating domains. Because the stress relaxation dynamics of artificial protein networks can be programmed at the genetic level (42), it will be interesting to explore the extent to which pattern formation can be further controlled by changes to protein sequence.

Acknowledgments: The assistance of Andres Collazo of the Biological Imaging Facility of the Beckman Institute at the California Institute of Technology is gratefully acknowledged. We thank David Tirrell for providing laboratory space, supplies and equipment. We also thank David Tirrell, Cole DeForest, John Bagert, and Kai Yuet for helpful discussions and suggestions during the preparation of the manuscript. This work was supported by grant number DMR-1506483 from the Biomaterials Program of the U. S. National Science Foundation.

Additional data is included online in the supplementary materials. 


\section{Supplementary Materials:}

Materials and Methods

Figures $\mathrm{S} 1$ to $\mathrm{S} 4$

Table S1

Movies S1 to S4

\section{References:}

1. F. S. Bates, Polymer-Polymer Phase-Behavior. Science 251, 898-905 (1991).

2. B. de Boer et al., Supramolecular self-assembly and opto-electronic properties of semiconducting block copolymers. Polymer 42, 9097-9109 (2001).

3. K. Sivula et al., Amphiphilic Diblock Copolymer Compatibilizers and Their Effect on the Morphology and Performance of Polythiophene:Fullerene Solar Cells. Adv. Mater. 18, 206-210 (2006).

4. G. B. Wei, P. X. Ma, Structure and properties of nano-hydroxyapatite/polymer composite scaffolds for bone tissue engineering. Biomaterials 25, 4749-4757 (2004).

5. H. Tanaka, Formation of Network and Cellular Structures by Viscoelastic Phase Separation. Adv. Mater. 21, 1872-1880 (2009).

6. H. Tanaka, Unusual Phase-Separation in a Polymer-Solution Caused by Asymmetric Molecular-Dynamics. Phys. Rev. Lett. 71, 3158-3161 (1993).

7. T. Taniguchi, A. Onuki, Network Domain Structure in Viscoelastic Phase Separation. Phys. Rev. Lett. 77, 4910-4913 (1996). 
8. W. W. Mullins, The Statistical Self-Similarity Hypothesis in Grain-Growth and Particle Coarsening. J. Appl. Phys. 59, 1341-1349 (1986).

9. H. Tanaka, Universality of viscoelastic phase separation in dynamically asymmetric fluid mixtures. Phys. Rev. Lett. 76, 787-790 (1996).

10. A. J. Wagner, J. M. Yeomans, Breakdown of Scale Invariance in the Coarsening of Phase-Separating Binary Fluids. Phys. Rev. Lett. 80, 1429-1432 (1998).

11. S. Tanaka et al., Pattern formation and coarsening during metastable phase separation in lysozyme solutions. Phys. Rev. E 65, 051804 (2002).

12. H. Tanaka, Y. Nishikawa, Viscoelastic Phase Separation of Protein Solutions. Phys. Rev. Lett. 95, 078103 (2005).

13. H. Tanaka et al., Network-forming phase separation of colloidal suspensions. J. Phys. Condens. Matter 17, L143 (2005).

14. S. Tanaka et al., Kinetics of phase separation and coarsening in dilute surfactant pentaethylene glycol monododecyl ether solutions. J. Chem. Phys. 135, 234503 (2011).

15. P. B. Rapp et al., Analysis and Control of Chain Mobility in Protein Hydrogels. J. Am. Chem. Soc. 139, 3796-3804 (2017).

16. L. J. Dooling et al., Programming Molecular Association and Viscoelastic Behavior in Protein Networks. Adv. Mater. 28, $4651-4657$ (2016).

17. V. N. Malashkevich et al., The crystal structure of a five-stranded coiled coil in COMP: A prototype ion channel? Science 274, 761-765 (1996).

18. B. D. Olsen et al., Yielding Behavior in Injectable Hydrogels from Telechelic Proteins. Macromolecules 43, 9094-9099 (2010). 
19. P. B. Rapp et al., Analysis and Control of Chain Mobility in Protein Hydrogels. J. Am. Chem. Soc. 139, 3796-3804 (2017).

20. D. E. Meyer, A. Chilkoti, Quantification of the effects of chain length and concentration on the thermal behavior of elastin-like polypeptides. Biomacromolecules $\mathbf{5}$, 846-851 (2004).

21. R. A. McMillan, V. P. Conticello, Synthesis and characterization of elastin-mimetic protein gels derived from a well-defined polypeptide precursor. Macromolecules 33, 4809-4821 (2000).

22. D. W. Urry, Physical Chemistry of Biological Free Energy Transduction As Demonstrated by Elastic Protein-Based Polymers. J. Phys. Chem. B 101, 11007-11028 (1997).

23. This complex phase behavior was sensitive to the detailed refolding procedure employed during protein purification of PEP (see Materials and Methods for details). We take this as further evidence that multiple, subtle interchain interactions influence the observed network LCST (cf. Fig. S2).

24. T. Luo, K. L. Kiick, Noncovalent Modulation of the Inverse Temperature Transition and Self-Assembly of Elastin-b-Collagen-like Peptide Bioconjugates. J. Am. Chem. Soc. 137, 15362-15365 (2015).

25. F. G. Quiroz, A. Chilkoti, Sequence heuristics to encode phase behaviour in intrinsically disordered protein polymers. Nat. Mater. 14, 1164-1171 (2015).

26. J. S. Haghpanah et al., Artificial Protein Block Copolymers Blocks Comprising Two Distinct Self-Assembling Domains. ChemBioChem 10, 2733-2735 (2009). 
27. C. B. Qian et al., Phase-Diagrams of Binary Polymer-Solutions and Blends. Macromolecules 24, 1655-1661 (1991).

28. E. L. Cheluget et al., Modifications of the Flory-Huggins-Goldstein Model for Accurate Description of Closed-Loop Phase-Diagrams. Chem. Eng. Sci. 48, 1415-1426 (1993).

29. S. K. Gunasekar et al., N-Terminal Aliphatic Residues Dictate the Structure, Stability, Assembly, and Small Molecule Binding of the Coiled-Coil Region of Cartilage Oligomeric Matrix Protein. Biochemistry 48, 8559-8567 (2009).

30. H. Tanaka et al., Universality of viscoelastic phase separation in soft matter. J. Phys. Condens. Matter 17, S3195-S3204 (2005).

31. H. Tanaka, Viscoelastic phase separation. J. Phys. Condens. Matter 12, R207-R264 (2000).

32. S. Song, J. M. Torkelson, Coarsening Effects on Microstructure Formation in Isopycnic Polymer Solutions and Membranes Produced via Thermally Induced Phase Separation. Macromolecules 27, 6389-6397 (1994).

33. Y. A. Li et al., Mobility of lysozyme inside oxidized starch polymer microgels. Soft Matter 7, 1926-1935 (2011).

34. P. Gribbon, T. E. Hardingham, Macromolecular diffusion of biological polymers measured by confocal fluorescence recovery after photobleaching. Biophys. J. 75, 10321039 (1998).

35. P. B. Rapp et al., Mechanisms of Diffusion in Associative Polymer Networks: Evidence for Chain Hopping. J. Am. Chem. Soc. 140, 14185-14194 (2018).

36. B. L. Sprague et al., Analysis of binding reactions by fluorescence recovery after photobleaching. Biophys. J. 86, 3473-3495 (2004). 
37. A. K. Gaigalas et al., Photodegradation of fluorescein in solutions containing n-propyl gallate. J. Phys. Chem. A 108, 4378-4384 (2004).

38. F. Stracke et al., Singlet molecular oxygen photosensitized by Rhodamine dyes: correlation with photophysical properties of the sensitizers. J. Photochem. Photobiol. A 126, 51-58 (1999).

39. H. R. Shen et al., Photodynamic crosslinking of proteins .2. Photocrosslinking of a model protein-ribonuclease A. J. Photochem. Photobiol. B 35, 213-219 (1996).

40. M. P. Sheetz, D. E. Koppel, Membrane Damage Caused by Irradiation of Fluorescent Concanavalin-A. Proc. Natl. Acad. Sci. 76, 3314-3317 (1979).

41. M. J. Davies, Singlet oxygen-mediated damage to proteins and its consequences. Biochem. Biophys. Res. Commun. 305, 761-770 (2003).

42. L. J. Dooling, D. A. Tirrell, Engineering the Dynamic Properties of Protein Networks through Sequence Variation. ACS Cent. Sci. 2, 812-819 (2016). 


\title{
Supplementary Materials for:
}

\section{Viscoelastic phase patterning in artificial protein hydrogels}

\author{
Peter B. Rapp, Bradley R. Silverman \\ correspondence to: peter.rapp@yale.edu
}

This PDF file includes:

Materials and Methods

Figs. S1 to S4

Tables S1

Captions for Movies S1 to S4

Supplementary References

Other Supplementary Materials for this manuscript includes the following:

Movies S1 to S4 


\section{Materials and Methods}

Protein expression and purification. Plasmids encoding the artificial proteins were transformed into BL21 or BL21 (DE3) chemically competent E. coli. After overnight culture, cells were inoculated (inoculation ratios of 1:50 - 1:100) into $1 \mathrm{~L}$ flasks containing Terrific Broth (TB) supplemented with $100-200 \mathrm{mg} \mathrm{ml}^{-1}$ ampicillin. Cells were grown to an $\mathrm{OD}_{600}$ of $0.8-1.0$ and then induced with $1 \mathrm{mM}$ final concentration of isopropyl $\beta$-D-1 thiogalactopyranoside (IPTG). After 4-5 h, bacterial cultures were harvested by centrifugation for 5-10 min at 10,000 , followed by lysis with $8 \mathrm{M}$ urea. Cell lysates were freeze-thawed at least once before being subject to high-power tip sonication for homogenization $(50 \mathrm{~mL}$ of lysate from a $1 \mathrm{~L}$ culture was typically treated with $30-50 \mathrm{~W}$ for $10 \mathrm{~min}$ in $0.5 \mathrm{~s}$ pulses). Homogenized lysate was clarified by high-speed centrifugation $(>30,000 \mathrm{~g}$ for $1 \mathrm{~h}$ ) and then subject to standard His-tag purification over Ni-NTA agarose beads (Qiagen) under denaturing conditions ( $8 \mathrm{M}$ urea).

Protein dialysis and refolding. Denatured, purified protein (ca. $1 \mathrm{mg} / \mathrm{mL}$ starting in $50-100 \mathrm{~mL} 8 \mathrm{M}$ urea plus phosphate buffer) was dialyzed against $4 \mathrm{~L}$ of distilled water at $4{ }^{\circ} \mathrm{C}$. The water was changed repeatedly $(5-6 \mathrm{X})$ over the course of several days. Typically, protein precipitation in the dialysate was used as the dialysis endpoint, after which point the aqueous suspensions were lyophilized. This procedure, which was used for all of the experiments reported here, routinely gave gels that displayed cloud points near $40{ }^{\circ} \mathrm{C}$ (cf. Fig. 1C). Notably, subtle changes to the refolding procedure (e.g., changes in dialysis temperature, starting buffer identity or dialysate exchange frequency) could both raise and lower the observed cloud-point temperature of PEP networks above or below $40{ }^{\circ} \mathrm{C}$. For example, frequent dialysate exchange of purified protein starting in urea plus Tris buffer gave protein batches that formed networks with undetectable cloud-points over the examined range $\left(25-95^{\circ} \mathrm{C}\right)$. We infer that the observed network LCST is not a simple function of the E midblock alone, but depends also on the folded state of the $\mathrm{P}$ endblocks, as well as on noncovalent interactions among E midblocks.

Hydrogel preparation. $100 \mathrm{mM}$ phosphate buffer ( $\mathrm{pH} 6.5$ - 7.4) was added to lyophilized PEP protein and the suspension was placed on ice for $2-4 \mathrm{~h}$ to promote gelation. Fluorescent hydrogels were prepared by adding low concentrations of labeled $\mathrm{PE}_{\mathrm{C}} \mathrm{P}$ to normal PEP networks (typically, $\mathrm{PE}_{\mathrm{C}} \mathrm{P}: \mathrm{PEP}$ mass ratios of 1:50 and 1:100 were used). Dye conjugation to cysteine-containing probes was performed as described previously (I).

Rheological analysis of gels. Oscillatory shear rheology was performed on 10\% (w/v) PEP hydrogels using an ARES-RFS strain-controlled rheometer (TA Instruments) equipped with a cone-and-plate geometry. The outer edge of the plate was coated with mineral oil in order to minimize evaporation from the exposed gel. Strain sweeps identified a linear regime between $0.1-10 \%$ strain at $10 \mathrm{rad} \mathrm{s}^{-1}$. Frequency sweeps were performed at a fixed strain amplitude of $1 \%$ between 0.01 and $100 \mathrm{rad} \mathrm{s}^{-1}$. Temperature data was collected at $1 \%$ strain and $10 \mathrm{rad} \mathrm{s}^{-1}$, at $5{ }^{\circ} \mathrm{C}$ intervals between $25^{\circ} \mathrm{C}$ and $60{ }^{\circ} \mathrm{C}$.

Cloud-point measurements. Protein solutions were prepared at concentrations ranging from 1 to $10 \%(w / v)$ in $100 \mathrm{mM}$ phosphate buffer, $\mathrm{pH} 6.5$ - 7.4. Solutions of PEP above $2-3 \%$ formed viscoelastic gels, whereas below $2 \%$ the solutions flowed easily. Solutions and gels 
were loaded between the two halves of a disassembled quartz cuvette. The cuvette was assembled by pressing the two halves together, which sandwiched the gel within a $0.1 \mathrm{~cm}$ thick cavity. Roughly $400 \mu \mathrm{L}$ of gel was required to fill the cuvette. The absorbance at 650 $\mathrm{nm}$ in response to temperature was monitored continuously on a Cary 100 Bio UV-Vis spectrometer equipped with temperature control. Gels were typically heated from $25^{\circ} \mathrm{C}$ to $95{ }^{\circ} \mathrm{C}$, held at $95{ }^{\circ} \mathrm{C}$ for $5 \mathrm{~min}$, then cooled to $5{ }^{\circ} \mathrm{C}$. The heating and cooling rates were held constant at $1-3{ }^{\circ} \mathrm{C} \mathrm{min}{ }^{-1}$, with minimal differences observed between the faster and slower rates. Prior to cloud-point measurements on the "EC" protein, a 5\% solution was placed at $4{ }^{\circ} \mathrm{C}$ on a rotator plate for $3-4$ days to promote oxidative crosslinking of the thiol groups.

Confocal microscopy, photobleaching and patterning. Imaging of spontaneous pattern formation and phase separation in labeled PEP hydrogels was performed on a Zeiss LSM 880 confocal microscope ( $488 \mathrm{~nm}$ with $10-20 \mathrm{X}$ objectives) equipped with a "Delta T" heated stage programmed to cycle between $25^{\circ} \mathrm{C}$ and $50^{\circ} \mathrm{C}$ within 60 seconds (Bioptechs, Butler PA). Labeled gels were placed on an ITO-coated, thermally conductive Delta T culture dish and sealed beneath a coverslip using $120 \mu \mathrm{m}$ Secure-Seal spacers (Life Technologies). Image analysis was performed in MATLAB. FRAP experiments were performed on a Zeiss LSM 880 equipped with a $25 \mathrm{~mW}$ Argon laser. Recovery curves were fit to an effective Fickian diffusion model using the MATLAB function nlinfit $(1,2)$.

Patterning experiments were performed on a Zeiss LSM 5 Exciter equipped with a $25 \mathrm{~mW}$ Argon laser (458, 488 and $514 \mathrm{~nm}$ ) and a $25 \mathrm{~mW}$ Diode (405 nm) laser. All laser lines, at maximum power, were typically activated during the photobleach. Bleach spot sizes ranged from $100-2000 \mu \mathrm{m}^{2}$. Bleaching of a $500 \mu \mathrm{m}^{2}(a=12.5 \mu \mathrm{m}$ radius $)$ circle at a scan rate of $4 \mu \mathrm{m} \mathrm{s}^{-1}$ required roughly 2000 scans to ensure efficient bleaching of fluorescein. Bleaching of larger spot sizes $\left(2000 \mu \mathrm{m}^{2}, a=25 \mu \mathrm{m}\right)$ was usually performed at a scan rate of $1 \mu \mathrm{m} \mathrm{s}^{-1}$. Typical bleaching times varied from $2-10 \mathrm{~min}$, depending on the size of the bleach spot and the total number of scans (2000 - 5000 scans). The total incident power emitted from the Argon laser during a typical fluorescein photobleach was measured to be $\sim 1 \mathrm{~mW}$ using a power meter. At $1 \mathrm{~mW}$ incident power, the average irradiance (power density) was estimated to be $50 \mathrm{~W} \mathrm{~cm}^{-2}$. After bleaching of the desired pattern, the sample was allowed to recover fluorescence for $15-30 \mathrm{~min}$. Gels were then slowly heated to 50 ${ }^{\circ} \mathrm{C}$ at rate of $2-3{ }^{\circ} \mathrm{C} \mathrm{min}^{-1}$ using a standard heated stage and an aluminum coverslip mount.

Oxidative crosslinking studies. Gels were prepared at 5\% in $100 \mathrm{mM}$ phosphate buffer $\mathrm{pH}$ 7 , supplemented with $25 \mu \mathrm{M}$ free fluorescein or Rose Bengal, with or without $100 \mathrm{mM}$ $\mathrm{NaN}_{3}$. Gels were sealed between two coverslips, separated by a 0.030 in sheet of PDMS. Sample irradiation was performed using a Coherent Innova $70 \mathrm{CW}$ Ar-Ion laser. The total incident beam power at $488 \mathrm{~nm}$ was fixed at $250-300 \mathrm{~mW}$ using a circular beam spot with a diameter of roughly $0.8 \mathrm{~cm}$. The irradiance (power density) was estimated to be 0.5 $\mathrm{W} \mathrm{cm}{ }^{-2}$. Gels were irradiated for $2 \mathrm{~h}$, then solubilized in $8 \mathrm{M}$ urea and crosslinking was assessed by SDS-PAGE under non-reducing conditions. 

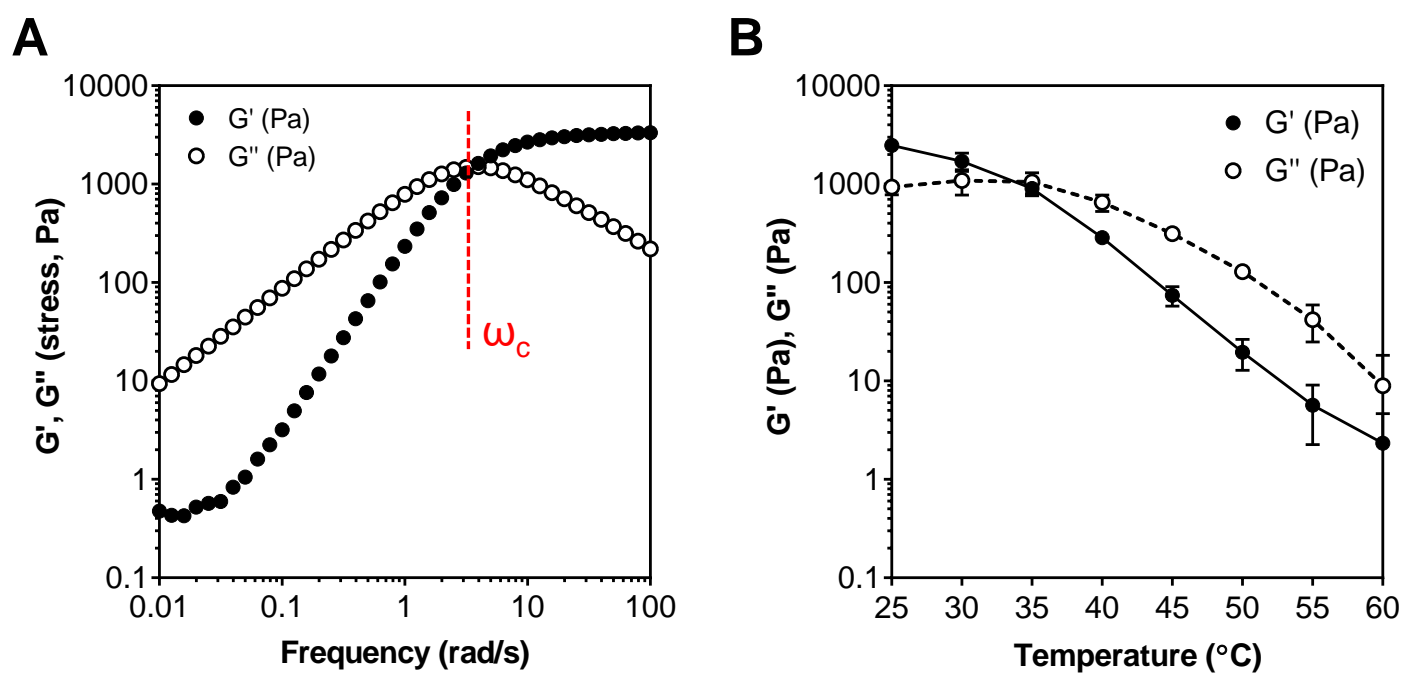

\section{Fig. S1}

Oscillatory shear rheometry of PEP hydrogels. (A) Frequency sweep of $10 \%(w / v)$ gels prepared in $100 \mathrm{mM}$ phosphate buffer at a fixed strain amplitude of $1 \%\left(25^{\circ} \mathrm{C}\right)$. The network behaves as a viscous liquid at low frequencies $\left(G^{\prime}<G^{\prime}\right.$ ') but transitions to elasticdominated behavior at high frequencies $\left(G^{\prime}>G^{\prime}\right.$ '). This transition occurs at a critical frequency $\omega_{\mathrm{c}}$, corresponding to the dominant stress relaxation mode of the physical network. (B) Storage $\left(G^{\prime}\right)$ and loss $\left(G^{\prime}{ }^{\prime}\right)$ moduli were measured for a $10 \%$ gel at $1 \%$ strain, $10 \mathrm{rad} \mathrm{s}^{-1}$ at various temperatures $(n=2$, mean $\pm \mathrm{SD})$. Although the viscous loss modulus dominates at high temperatures, weak crosslinking is still evident. 


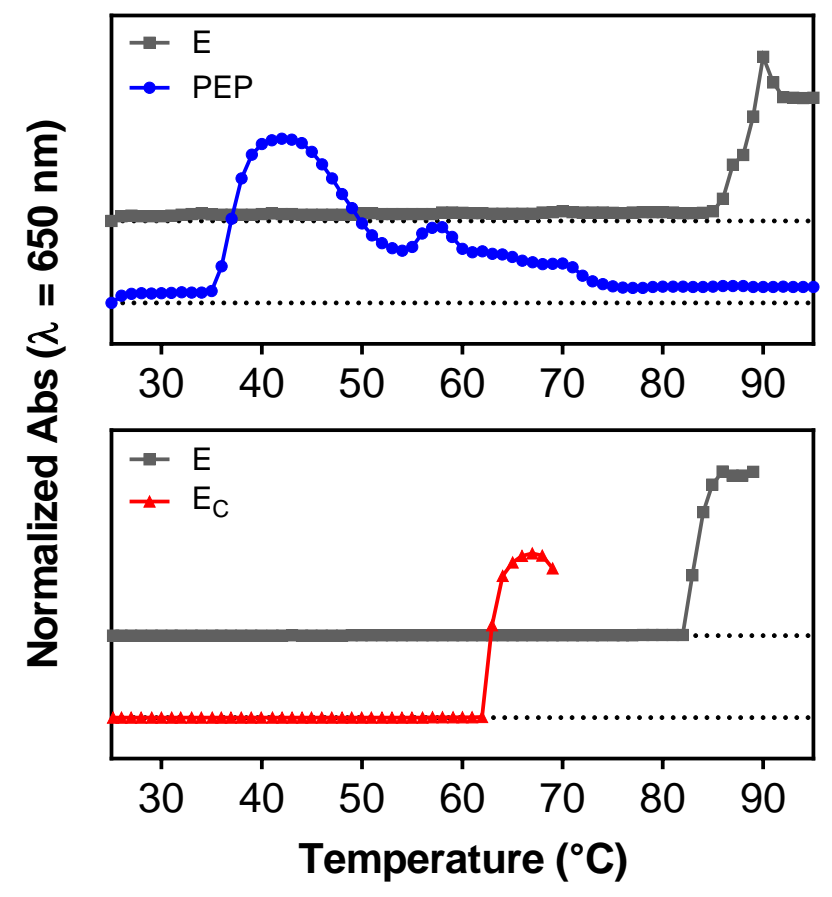

\section{Fig. S2}

Crosslinking affects the LCST of elastin-like proteins. Proteins were dissolved at a concentration of $5 \%$ in $100 \mathrm{mM}$ phosphate buffer (pH 6.5-7.4) and heated to $95{ }^{\circ} \mathrm{C}$ at a rate $1{ }^{\circ} \mathrm{C} \mathrm{min}-1$. The onset of turbidity was monitored at $650 \mathrm{~nm}$. (Top) The predicted LCST of $\mathrm{E}$ based on its repeat sequence $\left(70^{\circ} \mathrm{C}\right)$ is close to its observed transition temperature (80 $\left.-90{ }^{\circ} \mathrm{C}\right)$, whereas the transition temperature of gelled PEP is much lower $\left(38^{\circ} \mathrm{C}\right)$. (Bottom) The presence of an oxidized thiol (dimerization) in $\mathrm{E}_{\mathrm{C}}$ depresses its LCST by $\sim 20{ }^{\circ} \mathrm{C}$ relative to $\mathrm{E}$. 
A

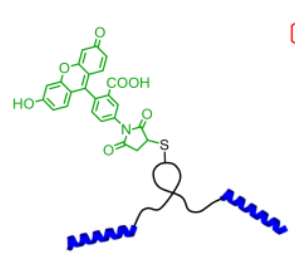

$P E_{C} P-f m$

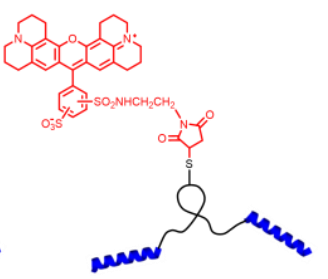

$\mathrm{PE}_{\mathrm{C}} \mathrm{P}-\mathrm{trm}$

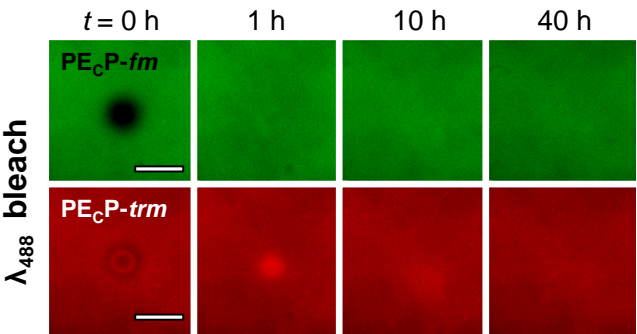

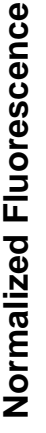

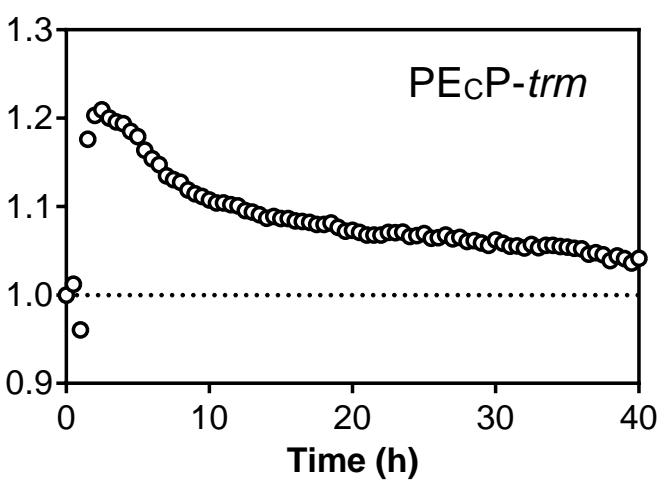

B
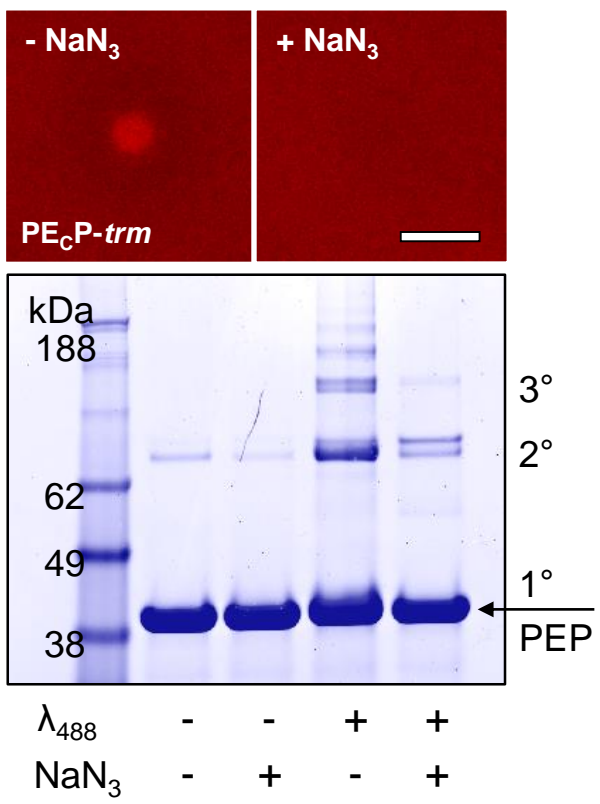

Fig. S3

Photobleaching promotes covalent interchain crosslinking and subsequent probe enrichment in bleach spots. (A) 5\% gels were labeled with green ( $\left.\mathrm{PE}_{\mathrm{C}} \mathrm{P}-\mathrm{fm}, 490 \mathrm{Ex} / 525 \mathrm{Em}\right)$ and red ( $\left.\mathrm{PE}_{\mathrm{C}} \mathrm{P}-\mathrm{trm}, 596 \mathrm{Ex} / 615 \mathrm{Em}\right)$ probes and photobleached (spot radius $a=25 \mu \mathrm{m}, \lambda_{488}$ bleach). Fluorescence recovery was monitored at $25{ }^{\circ} \mathrm{C}$. Red probes diffused into photobleached volumes and remained enriched for several hours. Scale bar $=100 \mu \mathrm{m}$. (B) (Top) Photobleaching promotes covalent interchain crosslinking by singlet oxygen generation. The presence of $100 \mathrm{mM} \mathrm{NaN}_{3}$, a strong singlet oxygen quencher, prevented red probe enrichment in $5 \%$ gels labeled with red and green probes $\left(\lambda_{488}\right.$ bleach). (Bottom) Bulk irradiation of $5 \%$ gels $\left(488 \mathrm{~nm}, 500 \mathrm{~mW} \mathrm{~cm}^{-2}\right)$ containing $25 \mu \mathrm{M}$ free fluorescein promoted covalent multimer formation of PEP chains. Multimerization was suppressed by the presence of $100 \mathrm{mM} \mathrm{NaN}_{3}$. Similar results were obtained replacing fluorescein with Rose Bengal, a highly efficient singlet oxygen generator. All scale bars $=100 \mu \mathrm{M}$. 

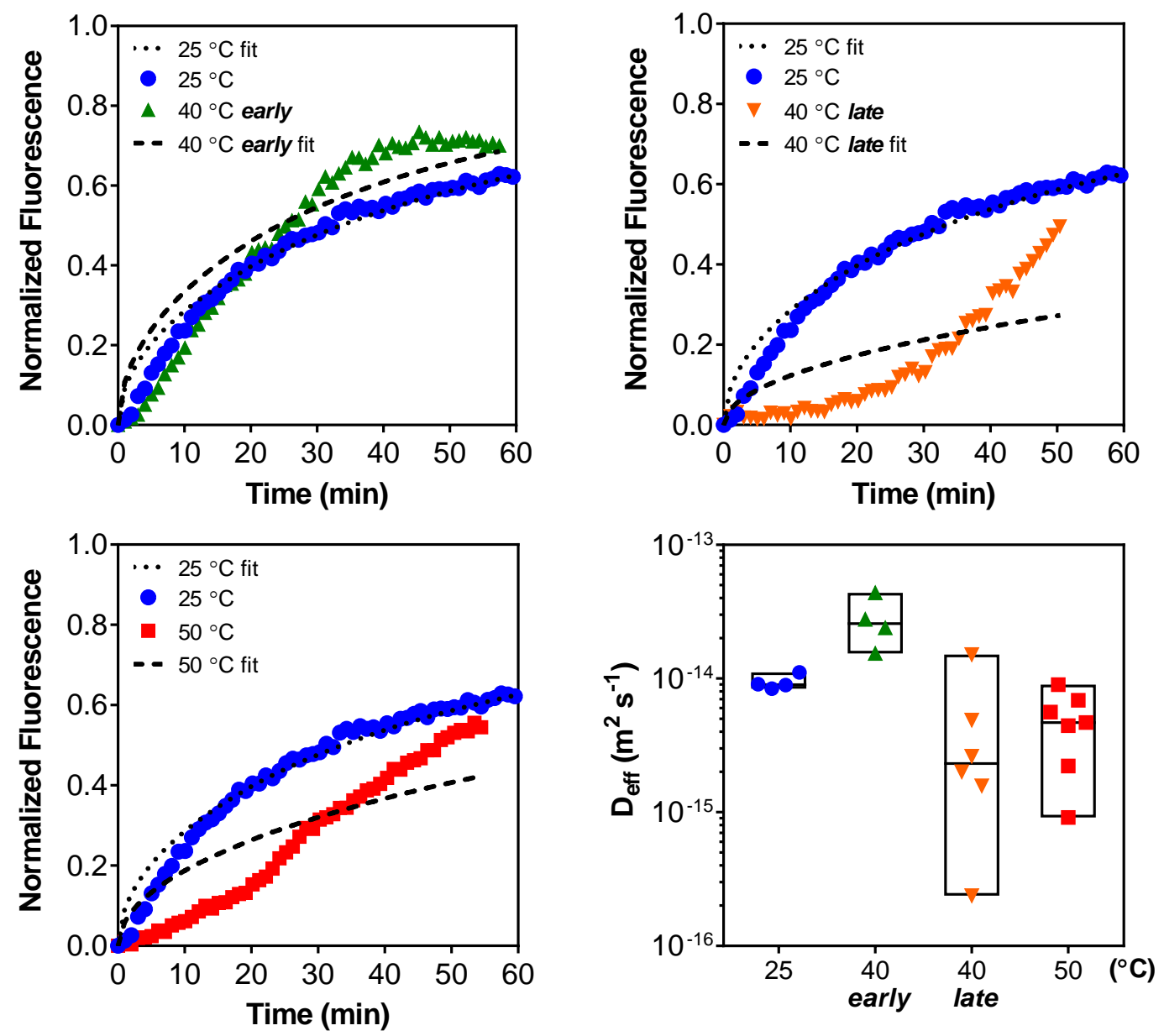

Fig. 54

Anomalous fluorescence recovery behavior above the LCST is poorly fit by standard FRAP models. Shown are representative FRAP traces acquired in a $10 \%$ PEP gel at $40{ }^{\circ} \mathrm{C}$ and $50{ }^{\circ} \mathrm{C}$, along with their corresponding fits assuming Fickian diffusion. Imprecision in estimating $D_{\text {eff }}$ at or above the LCST is attributable both to poor curve fitting as well as to the heterogenous character of the phase separated samples ( $n \geq 4$ per temperature; floating bars depict median as well as min/max estimates). 


\section{Table S1}

Plasmids and amino acid sequences for all artificial proteins described in the text. Protein coding sequences were confirmed by double-stranded DNA sequencing. Each "P" domain is highlighted in blue, and the " $\mathrm{E}$ " domain is highlighted in gray. Cysteine residues are in red.

\begin{tabular}{|c|c|c|}
\hline Plas & Protein & Molecular W \\
\hline 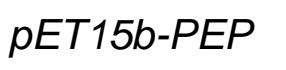 & PEP & \\
\hline \multicolumn{3}{|c|}{$\begin{array}{l}\text { MKGSHHHHHHHVDGSGSGSGSGSGSGAPQMLRELQETNAALQDVRELLR } \\
\text { QQVKEITFLKNTVMESDASGSGSGSGSGSGSGLDGHGVGVPGVGVPGVGV } \\
\text { PGEGVPGVGVPGVGVPGVGVPGVGVPGEGVPGVGVPGVGVPGVGVPGVG } \\
\text { VPGEGVPGVGVPGVGELYAVTGRGDSPASSAPIATSVPGVGVPGVGVPGE } \\
\text { GVPGVGVPGVGPGVGPGVGVPEGVPGGVPGGVPVGVGVGPG } \\
\text { EGVPGVGVPGVGVPGLLDGSGSGGSGSGSGPQMLRELQENAALQDV } \\
\text { RELLRQQVKEITFLKNTVMESDASGSGSGSGSGSGSGLEMHHHHHHK* }\end{array}$} \\
\hline$p E T 15 b-P E_{C} P$ & $\mathrm{E}_{\mathrm{CP}}$ & \\
\hline \multicolumn{3}{|c|}{$\begin{array}{l}\text { MKGSHHHHHHHVDGSGSGSGSGSGSGAPQMLRELQETNAALQDVRELLR } \\
\text { QQVKEITFLKNTVMESDASGSGSGSGSGSGSGLDGHGGVPGGVPGVGV } \\
\text { PGEGVPGVGVPGVGVPGVGVPGVGVPGEGVPGVGVPGVGVPGVGVPGVG } \\
\text { VPGEGVPGVGVPGVGELCYAVTGRGDSPASSAPIATSVPGVGVPGVGVPGE } \\
\text { GVPGVGVPGVGVPGGVPGGVGEGVGVGVGVGVGVGVGVGVG } \\
\text { EGVPGVGVPGVGVGGLDGSGGSGSSGSAPQMLRELQETNALQDV } \\
\text { RELLRQQVKEITFLKNTVMESDASGSGSGSGSGSGSGLEMHHHHHHK* }\end{array}$} \\
\hline$E 80 L-E$ & & \\
\hline \multicolumn{3}{|c|}{$\begin{array}{l}\text { GVGVPGVGVPGVGVPGEGVPGVGVPGVGVPGVG } \\
\text { /PGVGVPGVGVPGVGVPGEGVPGVGVPGVGELYA } \\
\text { IVPGVGVPGVGVPGEGVPGVGVPGVGVPGVGVPG } \\
\text { /GVPGVGVPGVGVPGEGVPGVGVPGVGVPGGLLE }\end{array}$} \\
\hline$p Q E 80 L-E_{C}$ & EC & 17,706 \\
\hline \multicolumn{3}{|c|}{$\begin{array}{l}\text { VGVPGVGVPGEGVPGVGVPGVGVPGVG } \\
\text { PGVGVPGVGVPGEGVPGVGVPGVGELYA } \\
\text { /PGVGVPGEGVPGVGVPGVGVPGVGVPG } \\
\text { GVPGVGVPGEGVPGVGVPGVGVPGGLLE } \\
\end{array}$} \\
\hline
\end{tabular}




\section{Movie S1}

Programmed emergence of a sponge-like morphology at $50{ }^{\circ} \mathrm{C}$ during early stage phase separation in a 10\% PEP hydrogel (related to Fig. 2). Total elapsed time 10 min. Scale bar $=100 \mu \mathrm{m}$.

\section{Movie S2}

A global view of spontaneous pattern evolution at $50{ }^{\circ} \mathrm{C}$ during late stage coarsening of PEP suggests a breakdown of self-similarity and the absence of a characteristic length scale (related to Fig. 2). Aqueous phase coarsening by droplet ripening and elastically hindered droplet coalescence are also clearly observed. Total elapsed time $16 \mathrm{~h}$. Scale bar $=1 \mathrm{~mm}$.

\section{Movie S3}

Viscoelastic "snapping" of a late stage coacervate tendril illustrates the persistence of viscoelastic coarsening mode at $50{ }^{\circ} \mathrm{C}$ (related to Fig. 2). Total elapsed time $1 \mathrm{~h}$. Scale bar $=50 \mu \mathrm{m}$.

\section{Movie S4}

Photobleaching enables transient patterning of non-equilibrium phase shapes during phase separation onset in a PEP hydrogel (related to Fig. 3). Shown is fluorescence recovery of a square photobleached pattern over $30 \mathrm{~min}$, followed by heating of the gel to $50{ }^{\circ} \mathrm{C}$ for 1.5 $\mathrm{h}$. Heating matures the photobleached region into a cylindrically patterned coacervate domain. Total elapsed time $2 \mathrm{~h}$. Scale bar $=50 \mu \mathrm{m}$.

\section{Supplementary References}

1. P. B. Rapp et al., Analysis and Control of Chain Mobility in Protein Hydrogels. $J$. Am. Chem. Soc. 139, 3796-3804 (2017).

2. B. L. Sprague et al., Analysis of binding reactions by fluorescence recovery after photobleaching. Biophys. J. 86, 3473-3495 (2004). 


\section{Other files}

\begin{tabular}{lc} 
movieS1.mov $(2.46 \mathrm{MiB})$ & view on ChemRxiv • download file \\
\hline movieS2.mov $(9.87 \mathrm{MiB})$ & view on ChemRxiv $\circ$ download file \\
\hline movieS3.mov $(97.45 \mathrm{KiB})$ & view on ChemRxiv $\circ$ download file \\
\hline movieS4.mov $(7.03 \mathrm{MiB})$ & view on ChemRxiv $\circ$ download file
\end{tabular}

